1 Sabanathan S, Richardson J, Mearns AJ. Management of pain in thora pain in thoracic surgery. Br f Hosp Med 1993; 50: 114-20. Alden Press, 1988.

McGee J O'D, Isaacson PG, Wright NA. Oxford textbook of

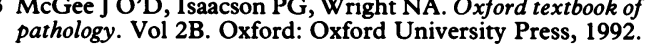

4 Werbel SS, Ober KP. Acute adrenal insufficiency. Endocrinol Metab Clin N Am 1993; 22: 303-27.

5 Kehlet parent A rational approach to dosage and preparation of garenteral grocedure. Acta Anaesth Scand 1975; 19: 260-4.

\title{
Methotrexate-induced pericarditis and pericardial effusion; first reported case
}

\author{
LN Forbat, BW Hancock, AH Gershlick
}

\begin{abstract}
Summary
We report a case of methotrexateinduced pericarditis and pericardial effusion in a 22-year-old pregnant woman. These complications have not previously been described as isolated phenomena associated with methotrexate therapy.
\end{abstract}

Keywords: pericarditis, pericardial effusion, methotrexate, toxicity

\section{Introduction}

Methotrexate is used in different schedules in oncology and commonly in low dose oral regimens in rheumatology. The recognised features of methotrexate toxicity are shown in the box. Pericarditis and pericardial effusion have not previously been described as isolated phenomena, whereas pneumonitis and/or pleurisy are complications which occur idiosyncratically ${ }^{1}$ in patients treated with methotrexate $e^{2,3}$ and are reported to occur in $5-12 \%$ of treated patients. ${ }^{1,4}$ In cyclical regimens used in oncology, pleural/pulmonary complications are very rare; however, in patients with persistent gestational trophoblastic disease receiving low-dose intramuscular repeated-course schedules, over $20 \%$ of patients may be affected. ${ }^{5}$ Steroids are not usually used to treat methotrexate lung; however, they may be of therapeutic value in some patients. ${ }^{1}$ Occasionally the symptoms may be so severe and intractable that a change in cytotoxic therapy is required. We report a case of pericarditis and pericardial effusion leading to symptoms of breathlessness.

Cardiology, Glenfield NHS Trust, Leicester, UK

AH Gershlick

Correspondence to Dr LN Forbat, Locum Consultant Physician and Cardiologist, Department of Medicine, Good Hope Hospital NHS Trust, Rectory Road, Sutton Coldfield, West Midlands B75 7RR, UK

Accepted 16 November 1994 atrophy, nephropathy, defective
oogenesis/spermatogenesis, osteoporosis, CNS various effects following intrathecal treatment

\section{Case report}

A 22-year-old woman presented with a molar pregnancy. Beta-human chorionic gonadotrophin (BHCG) levels were 6295 IU/1 (normal $<7$ ) and a complete hydatidiform mole was removed at dilatation and curettage. Postoperatively BHCG levels fell to $3720 \mathrm{IU} / 1$; however two months later a computed tomography (CT) scan showed a single pulmonary nodule and her BHCG levels remained elevated. She was treated with intramuscular methotrexate and folinic acid rescue. ${ }^{6}$ Nine courses were given over a 16-week period (total methotrexate dose $1.8 \mathrm{~g}$ ). In month two she presented with pleuritic chest pains of the left and anterior chest. She was otherwise well and had no cough, sputum, or fever. On examination the only abnormality was a pleural rub at the left base. Methotrexate-associated pleurisy and pneumonitis was diagnosed. She was advised to ensure adequate hydration and symptoms responded to treatment with naproxen. BHCG levels remained normal during this episode. Two months after treatment she had recurrence of her symptoms of pleuritic chest pain and was also breathless on exertion. She remained afebrile and on clinical examination there were no abnormal signs. Chest X-ray showed cardiomegaly and echocardiogram revealed a large pericardial effusion $(5 \mathrm{~cm}$ depth posteriorly and $2 \mathrm{~cm}$ anteriorly) without evidence of impaired myocardial function at rest. ECG, full blood count (eosinophil count $0.1 \times 10^{9} 1^{-1}$ ), urea, electrolytes and liver function were normal. Erythroyte sedimentation rate was $18 \mathrm{~mm} / \mathrm{h}$. Pericardial aspiration was performed and $650 \mathrm{ml}$ of clear fluid was drained. Cytology revealed abundant lymphocytes, reactive mesothelial cells and scattered eosinophils, but no malignant cells. Biochemistry of the aspirate was consistent with an inflammatory exudate; glucose $4.7 \mathrm{mmol} / 1$ (serum 5.2), albumin $28 \mathrm{~g} / 1$, total protein $49 \mathrm{~g} / \mathrm{l}$. Bacterial cultures including tuberculosis were negative, as were serial viral titres and an auto-antibody screen. Her symptoms of breathlessness resolved and there has been no recurrence after 18 months. 


\section{Discussion}

This is the first reported case of methotrexateinduced pericarditis and pericardial effusion. The syndrome of methotrexate pleural toxicity is invariably of pleuritic pain with normal chest $\mathrm{X}$-ray and ventilation/perfusion scan. X-Ray changes if present are non-specific and diagnosis may require bronchoscopy and lavage ${ }^{3,7}$ or lung biopsy. ${ }^{7}$ The differential diagnosis is infection, pulmonary infarction and tumourrelated complications. Pleural effusions after methotrexate therapy are most likely to be due to cancer; in this case the fluid should be drained and therapy changed. This patient had methotrexate chemotherapy followed by two episodes of pleurisy, the second being associated with pericarditis and a large pericar-

1 Massin F, Coudert B, Marot JP, Foucher P, Canus P, Jeannin L. Methotrexate pneumonitis. Rev Mal Respir 1990, 7: 5-15

2 Twohig KJ, Matthay RA. Pulmonary effects of cytotoxic agents other than bleomycin. Clin Chest Med 1990; 11: $31-54$.

3 Lehne G, Lote K. Pulmonary toxicity of cytotoxic and immunosuppressive agents; a review. Acta Oncol 1990; 29: 113-24.

4 Biggs JC, Atkinson K, Avalos BR, et al. A multi-institutional study of Bucy 2 followed by allogeneic marrow transplantation in CML. (meeting abstract). Exp Hematol 1991; 19: 572. dial effusion. As in the majority of cases with pleural toxicity there was no peripheral eosinophilia; however, eosinophils were present in the pericardial fluid. An unrecognised viral infection or a reaction to an unsuspected allergen remains a possible cause; however, we believe that the pericardial effusion was most likely a complication of methotrexate chemotherapy and that, as with methotrexate pneumonitis, this is likely to be an immunemediated serositis. ${ }^{7}$ The possibility of pericarditis and significant pericardial effusion should be considered as a potentially severe complication of methotrexate therapy. The regular use of echocardiography could provide information on the incidence of isolated asymptomatic pericardial effusion and that concomitant with methotrexate pneumonitis/pleurisy.

5 Sheridan E, Hancock BW, Smith SC, et al. Gestational trophoblastic disease: experience of the Sheffield (United Kingdom) supraregional screening and treatment service (Review). Int F Oncol 1993; 3: 149-55.

6 Rustin GJS. Trophoblastic diseases. In: Shaw R, et al. Gynaecology. Edinburgh: Churchill Livingstone 1992; pp 562-4.

7 Akoum GM, Maynaud CM, Touboul JL et al. Use of bronchoalveolar lavage in the evaluation of methotrexate lung disease. Thorax 1987; 42: 652-5.

\title{
The association of hereditary neuropathies and heritable skeletal disorders
}

Royal Hallamshire Hospital, Glossop

Road, Sheffield

S10 2JF, UK

Department of

Clinical

Neurophysiology

AK Chattopadhyay

RH Kandler

Department of

Neurology

B Sharrack

Accepted 16 November 1994

\author{
AK Chattopadhyay, RH Kandler, B Sharrack
}

\begin{abstract}
Summary
We describe two patients with associations of hereditary neuropathies and heritable skeletal disorders not previously reported. The first patient had Marfan's syndrome and hereditary motor and sensory neuropathy Type 1 . The second patient had Ehlers-Danlos syndrome, Klippel-Feil syndrome and tomaculous neuropathy.
\end{abstract}

Keywords: hereditary neuropathy, heritable skeletal disorders, Marfan's syndrome, Ehlers-Danlos syndrome, Klippel-Feil syndrome

There are occasional reports of the association between a peripheral neuropathy and Marfan's syndrome, Ehlers-Danlos syndrome, or Klippel-Feil syndrome. ${ }^{1-5} \mathrm{We}$ report two patients with associations not previously described.

\section{Patients}

Case 1

A 19-year-old man presented with progressive difficulty in walking. He had no sensory symptoms. He was of thin build. His paternal grandmother, father and sister were said to have a similar build. On examination, he had bilateral pes cavus and decreased tendon reflexes. He also had arachnodactyly, a high arched palate and bilateral lens dislocations. A diagnosis of Marfan's syndrome and a probable hereditary neuropathy was made.

Echocardiography showed mitral valve prolapse. Nerve conduction studies showed marked slowing of motor conduction velocity in the median nerve $(15 \mathrm{~m} / \mathrm{s}$; lower limit of normal $48 \mathrm{~m} / \mathrm{s}$ ) and absence of sensory action potentials (median, ulnar, radial and sural). Electromyographic examination showed neuropathic changes in tibialis anterior. The findings thus showed a severe demyelinating and degenerating neuropathy consistent with hereditary motor and sensory neuropathy Type I.

Other family members were not available for clinical or electrophysiological examination.

\section{Case 2}

A 16-year-old boy presented with tingling in the distribution of the median nerve in the right hand. He had previously complained of tingling in the little finger of the left hand which was subsiding. On examination, he had blunting to pin prick sensation in the right median 